\title{
A Teacher's Perspective on Technology in the Classroom: Computer Visualization, Concept Maps and Learning Logs
}

\author{
Charles L. Hurwitz and \\ Gerald Abegg \\ $\propto$
}

THE USE OF COMPUTER SOFTWARE IN THE CLASSROOM HAS CHANGED THE WAY that a teacher can approach chemistry education at the high school level. Through the use of concept maps, electronic learning logs, and computer simulations, students can be provided a deeper understanding of chemistry and research methods than previously possible, while avoiding rote learning. At the same time, the teacher can engage in on-going formative assessment of the class's progress. We report on the methods that we have studied which support these changes in the classroom.

\section{Introduction}

When we discussed the ideas for this special theme edition with the editor, we recommended that an article should be included that examined the

Charles Hurwitz has taught high school chemistry for over twenty years. For the past ten years he has been a consultant on multiple National Science Foundation funded projects for the design of computer software for science education. He is currently completing his EdD at Boston University with a study of how the intervention of such software can affect student learning.

Gerald Abegg is Professor Emeritus of Science Education at Boston University. Over his more than 25 years at Boston University he has directed numerous NSF sponsored curriculum development and teacher education programs focused on the improvement of science education $\mathrm{K}-12$. 
impact of computer technology on the teacher and students in the classroom setting. Our reasons for this recommendation were based on our experience of seeing computers introduced into selected schools and classrooms more than twenty five years ago and then seeing their use virtually explode as the technology of the computer expanded. From the primitive stages of the four megahertz PET and the Apple II with tape recorders as data input/output to the current gigabyte, 500 megahertz desktop machines with CD ROM and multi-media capabilities, the growth of the technology has led to a variety of educational uses-some excellent and some which do not utilize the power of the technology.

The initial introduction of computers in the schools focused on those tasks which were mundane, repetitive or record keeping. Thus, it was not surprising to see the early computer use in the administrative offices and in specialized computer labs where students could engage in fairly routine drill and practice tasks. Early computers with limited memories functioned as modest calculators with the capacity to handle simple spreadsheets and a variety of programmed instruction style materials. With the introduction of more powerful computers, some teachers and their students began to explore different ways of doing many of the instructional tasks, which are part of the teaching-learning process. The use of larger spreadsheets for data analysis and the development of simulations began to appear in selected classrooms.

Although there continues to be a time lag between software development and the hardware, the lag was substantially reduced by the early 90s when the personal computer began to reach a large number of schools and homes. The adoption of these more powerful computers as educational tools has had a profound impact on many aspects of education because of the multifaceted range of materials which became accessible to the school and classroom environment. The literature is filled with articles which express opinions, research data and policy studies documenting the pros and cons regarding the introduction of computers in the schools. In the remainder of this article we discuss the impact of computer technology over the past decade on one classroom. We examine this impact from the perspective of conceptual change research since that is the theoretical foundation of much of our work with students.

The two authors have collaborated these past ten years on a several research projects. While each of us has had a personal set of experiences, for this paper we are sharing our experiences and analyses with a single voice. 


\section{Our Background}

Over the past decade we have participated in National Science Foundation sponsored science education research projects aimed at understanding how students learn science, and how to use computer technology for this purpose. The projects in which we participated that had the greatest impact on the use of computers in our classroom are: On Growth and Form (OGAF, National Science Foundation grant number REC 9112301), and Water and Molecular Networks (WAMNet, National Science Foundation grant number REC 9253407) at Boston University under Professor H. Eugene Stanley; and Quantum Science Across Disciplines (QSAD, National Science Foundation grant number REC 9554198) also at Boston University under Professor Peter Garik. In addition, we participated in summer institutes for a teacher enhancement program, which grew out of the On Growth and Form project. This project, Patterns in Nature (PINS, National Science Foundation grant number ESI 9353500), Professors Gerald Abegg and H. Eugene Stanley co-principal investigators, brought teachers to Boston University to introduce them to the science of how patterns develop in dynamic systems. The content and methods of these workshops relied heavily on computer-based instruction.

Beginning with the OGAF project, we found ourselves immersed in a new way of understanding chemistry and chemical dynamics. Our own training in chemistry and our teaching careers began more than a decade before computers became common in chemistry research and instruction. The ability of the computer to provide a visual representation of the outcome of a model for chemical dynamics changed our way of thinking about scientific theory, the meaning of simulations, and the methods of chemistry instruction. Suddenly, we were presented with a tool that allowed us to investigate the outcomes of chemistry experiments, whether they were actually within the range of classroom laboratory equipment or not.

What was most thrilling for us was the ability to use the computer to realize models of behavior. With the introduction of the computer, we found ourselves in a new scientific wonderland. No longer were we bound to experiences on the human scale. With computer modeling we could experiment with phenomena on scales that were very small, or very large! Or very short, or very long! And we were empowered with the ability to perform gedanken experiments - the mental exercise of supposing everything were as simple as just relying upon a small set of parametersthrough the use of simulations. 
To be specific, a common chemical process is to coat one metal with another metal electrochemically. The process involves charging a surface and attracting to it atoms of the opposite charge from solution. Thus, to coat a surface with copper atoms, we would place the surface in a bath of copper sulfate (say), and charge the surface negatively. Positive copper atoms in solution would then be attracted to this surface.

At the outset of the OGAF project we performed such an electrodeposition experiment using copper. We learned that the visually apparent dendritic growths that developed directly reflected the underlying microscopic processes that occurred in the reaction (Figure 1).

To study these processes, coupled with the wet lab experiment, we used computer programs to simulate the growths that we observed. These simulations only included a fraction of all the processes that were present in the actual experiment (Figure 2). Nevertheless, the visual outcome of the computer simulation was remarkably similar to the experiment. This left us with new insights into the limitations and meaning of scientific models and theories, and the role that computer models play in the sciences and can play in the classroom.

Ten years have elapsed since then. During the intervening time we have worked hard to incorporate computer-based instruction into the classroom using inquiry-based learning and constructivist approaches based on conceptual change theory. The succeeding projects with which

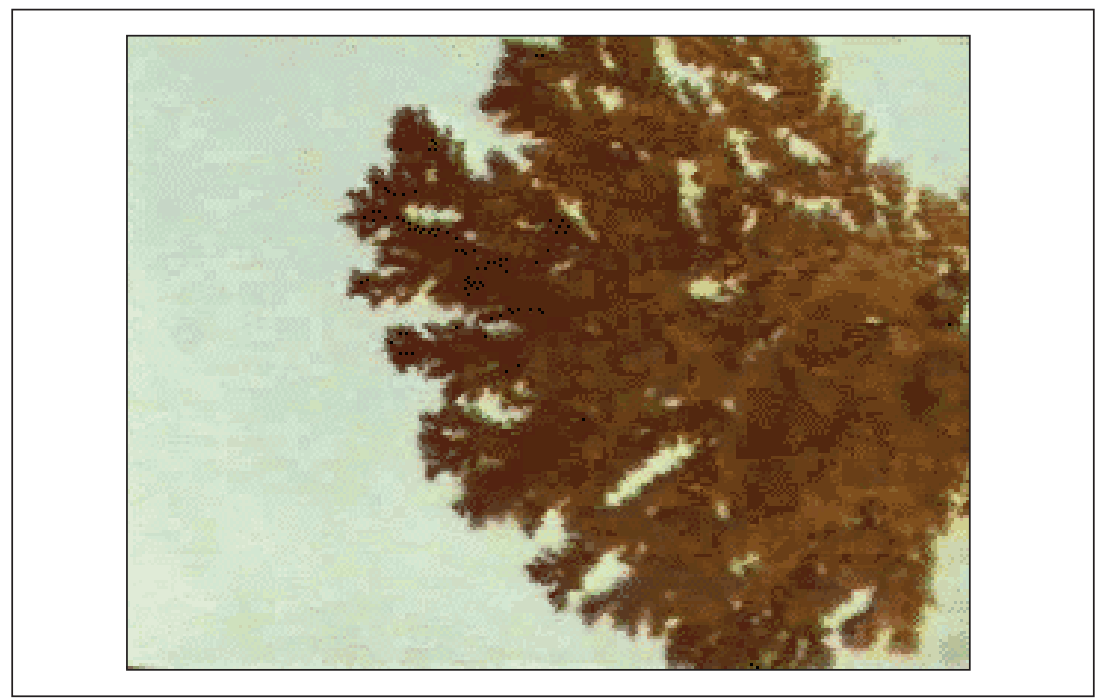

Figure 1. View of laboratory experiment: Electrochemical Deposition 


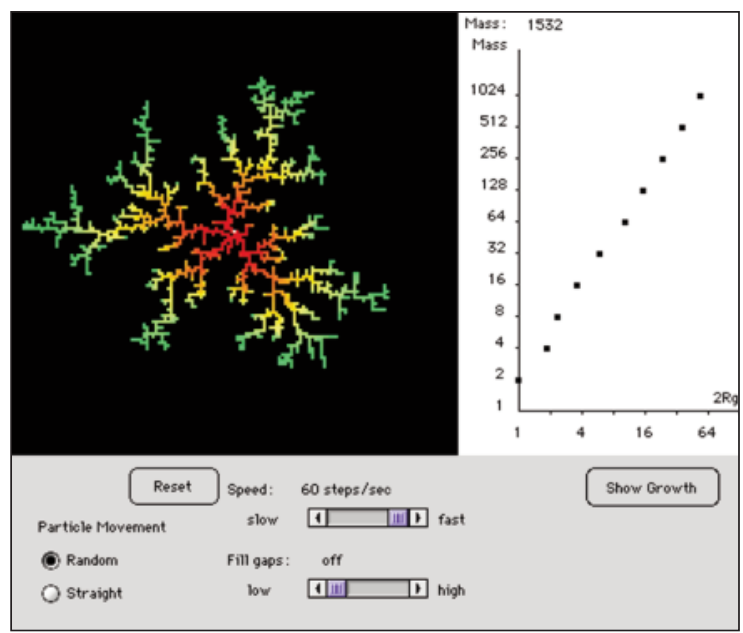

Figure 2. Computer simulation for aggregate growth by diffusion: An incoming particle moves up, down, right or left randomly until it touches the growing aggregate. Upon contact it sticks and a new particle begins the same random walk from an arbitrary point surrounding the aggregate.

we worked, WAMNet and QSAD, used computer simulations to model more common chemical behavior. During the process of integrating these computer-based materials into our classroom we have discovered the synergy between them and the inquiry-based learning and constructivist methods called for by the National Research Council in the National Science Education Standards (National Research Council, 1996).

For example, in teaching about gases, the traditional model is that of the ideal gas. But what is an ideal gas? How does it differ from a real gas? Why should we even attempt to apply the results of reasoning about an ideal gas to actual gases in the laboratory? If there is no link offered between the notion of the ideal gas and the real gas, if the student is never educated in the connection between models of behavior and experiments, then how can a student ever develop an intuition for chemical behavior?

In the WAMNet computer simulation it was possible to alter parameters in an interactive computer simulation so that students could vary gas behavior from that of the ideal gas to that of one with interacting molecules. By this means, students could discover the range of temperature and density over which the gas behaved ideally, and the cutoffs beyond which non-ideal behavior occurred (Figure 3). This investigation provided students with the bridge between the model and the real world, and is a mechanism for building chemical intuition for gas behavior. 


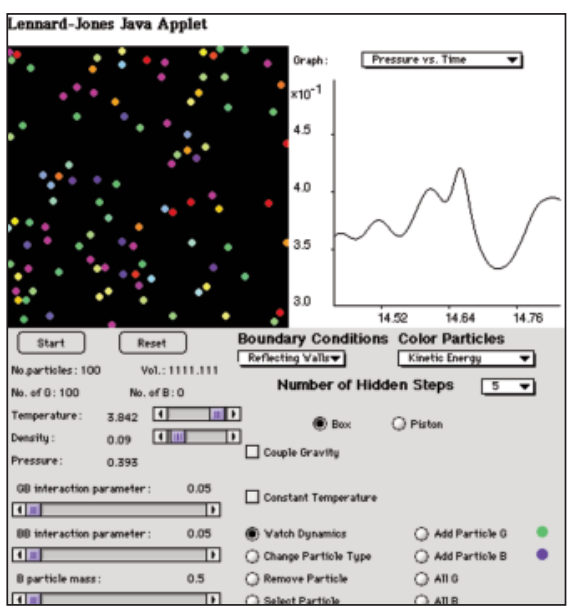

Figure 3. Simulation of atoms in a gas: by varying density and temperature, the user can observe the change in pressure of the gas.

\section{The School Setting and Our Beliefs About Teaching Chemistry}

The school system in which this work has been pursued is very supportive of innovative instructional methods. We received encouragement towards our goal of using computer-based technology in our classroom. One of us $(\mathrm{CH})$ has been allowed to substitute off-campus computer and technology enrichment activities for regular school duties. In addition, the school provided space for the computers for the WAMNet project.

The student audience we have been addressing is drawn from a suburb of Boston. Between 85 percent and 90 percent of the school's students go on to four-year colleges. The per capita income of the town is $\$ 28,300$ and 51.8 percent of the town's residents have at least a bachelors degree. Student and parental expectations of academic success are high. Half of the honors students in chemistry take the SAT II in Chemistry, and almost all score higher than 600. Most of the students in honors chemistry are sophomores who have taken physics as freshmen.

As we see it, the goal of a chemistry course is to help the student develop chemical intuition relating to whether a reaction will occur and what the energy involved in such a reaction is. Such an intuition about chemistry must be based on an understanding of chemical processes and structure. If we are successful in our instruction, then at the conclusion of our course the student is prepared to provide speculative answers to 
questions that arise about what he or she observes in the real world. A key objective to achieve this goal of chemical intuition is to provide students with an understanding that conclusions can be drawn about macroscopic experiments and observations by reasoning about microscopic behavior. The computer provides an important bridge here between microscopic theory and macroscopic behavior, which we were not able to offer the student prior to the introduction of interactive visual simulations.

Our view of computers in the classroom is that they provide the means for students to investigate scientific models along the lines that scientists do. It is our experience that it is common for the chemistry teacher to discover that students who have performed a wet laboratory do not recognize the connection between their experimental observations and their textbook/lecture instruction. Wet labs are critical to chemistry, and more generally to science education. They provide the examples around which we hope our students will build their intuition. However, if the associated scientific model for explaining the experimental behavior is not learned, then the transfer to later examples will be difficult or nonexistent. It is here that we have been seeking to exploit the power of interactive computer visualization, as in the example above about ideal gas laws.

\section{Inquiry-Based Learning as a Pedagogical Strategy}

Both the National Standards and the Massachusetts State Standards call for inquiry-based learning in the teaching of the sciences. Despite this fact, students arrive in our classroom unfamiliar with inquiry-based learning, and in many instances resistant to it. Moreover, as teachers we are confronted with the fact that inquiry-based learning is an objective for classroom activity; it is not a complete system for bringing the student to a new conceptual understanding or model of scientific phenomena.

The model closest to our instructional strategy for integrating inquiry-based learning into the classroom is that of Hewson and Hewson (1983). In turn, their model is based on a theory of conceptual change provided by Posner et al (1982). This theory proposes that for a student to adopt a new conceptual model, he or she must pass through four stages of change. First, the students have to become dissatisfied with their current model of events. Second, the new model must explain the discordant events where the original model could not. Third, the new model must be understandable, that is, it must be within the cognitive range of the student. Finally, the student should be aware of the usefulness of the new scientific 
model. These stages are referred to as dissatisfaction, plausibility, intelligibility, and fruitfulness by Hewson and Hewson (1983).

Our experience is that when students begin their study of chemistry they are filled with alternative conceptions and misconceptions about the functioning of the physical world. Some of these conceptions apparently are based upon naive knowledge and interpretation of personal experience. In other instances, we find that students arrive in introductory chemistry with misconceptions fostered by earlier instruction. Finally, throughout the course we teach, students are constantly generating conceptions based on their interpretation of the material we present. Oftentimes, these interpretations are based upon prior misconceptions, or misinterpretations and hypothesizing not intended by the curriculum. As a result, when students engage in selected inquiry-based exercises, especially using self-generated questions or questions they generate after an initial prompt from the instructor, conflicts between their own scientific conceptions and data quickly emerge.

While these conflicts may be with empirical data, computer simulations can be especially effective in confronting students with their misconceptions, or incomplete realizations, when the problems are in dealing with models of the microscopic, which are abstract, intangible, and invisible.

For example, students frequently enter first year chemistry with a strong background in the Bohr model of the atom. In the Bohr model, electrons orbit the nucleus at fixed radii in a manner akin to the way that planets circle the sun. While the Bohr model was a significant breakthrough in the history of physics and chemistry, its usefulness lasted about ten years before inconsistencies with experimental data drove theorists to replace it with the modern theory of quantum mechanics.

Specifically, quantum theory predicts the shape of the regions of space occupied by electrons near the nucleus. One of these shapes is the "p-orbital." Its appearance is very much like that of a dumbbell, and not at all like that of a circular orbit. Confronted by this discrepancy on the computer screen, for such objects are abstract and impossible to directly observe, students who cling to the Bohr model are quickly confounded by the discrepancy between the two theories. It is incidents like this that we have observed in our class that produce the dissatisfaction called for by the conceptual change theory.

Another example of the power of the computer to confront a student with a misconception is provided by our experiment using the WAMNet program to verify Dalton's Law of Partial Pressure. The students were 
required to present their procedure to the instructor. Since the students could maintain control over a variety of thermodynamic variables, just how they proved that the total pressure was equal to the sum of the partial pressures of the individual gases could vary. When their procedure was approved, the students were warned that as they collect data it would be wise to check the validity of the results.

One student approached the teacher and anxiously reported that no matter what she did she could not match her data with the Ideal Gas Law. The teacher responded supportively inquiring about the range of parameters she was using, and asking her to tell him how her data was indeed behaving. This alone did not resolve the issue. The teacher and student then went to the computer screen to observe the simulation. The computer program was simulating the behavior of the gas through the use of disks representing the atoms. Ideal gas behavior is valid at low densities. Low density is represented on the computer screen by the presence of only a few atoms in a given view area. What the teacher observed was that indeed the student was conducting the simulation with only a few disks. However, the screen displayed relatively large disks for the atoms. With this in mind, the teacher asked, in an off-hand manner, for the student to describe what she was seeing on the screen. Enlightenment hit her as she realized that large radii implied a high density even if there were only a few atoms present. An experience like that teaches a student more about the concepts of physical behavior than simply satisfying an equation!

Experiences like these have us convinced that computer-based inquiries can promote student learning and reconceptualization. However, the initial impetus of recognizing a discrepant event by itself may not always translate into learning. If a new concept is not provided for the student, the discrepant event may well be ignored and the old concept retained. Indeed, this is the way that the scientific community itself behaves (Kuhn, 1970). In our classroom we have found that to help the students construct new knowledge we must provide them with closure to this conflict by offering a new theory which is intelligible, plausible and fruitful (Posner et. al., 1982).

The intelligibility of the new theory relies upon the insightfulness and communication skills of the instructor and the authors of the supporting materials and software.

The plausibility of the new concept must be made by the instructor through associated examples demonstrating consistency of the new theory with prior empirical and conceptual knowledge. From our experience, this 
task is the most difficult step in the conceptual change process. As instructors, we have had to rely upon our pedagogical knowledge to convince the students of this plausibility by providing multiple examples and explanatory metaphors. Moreover, when students work in teams, the team must collectively arrive at the conclusion that the new theory is indeed plausible through arguments within the group. Sometimes teams will arrive at incorrect conclusions, indicating that intelligibility has not been achieved. We assess this through their learning logs (described below) and attempt to channel their discussions through probing questions, which rely upon conflicts between the misconception and the empirical or simulation-based data.

Where we have found the power of computer simulations to be greatest is in offering students the opportunity to test the fruitfulness of their new conceptions. Prior to our use of computer simulations, we could rarely directly address the issue of fruitfulness since experimental limitations make it difficult for students to test application of new models to a broad range of experimental parameters. By contrast, with the computer, students can pursue the application of a new model to nearly arbitrary parameters. As the instructors, we of course provide students with initial suggestions for inquiries to validate the fruitfulness of the model. Frequently, the visual display of the outcome of such an inquiry will be unexpected. However, using the new model to provide an explanation helps convince the student of the validity of their new knowledge.

\section{Logistics of Using Computers in the Science Classroom}

From our experience in the classroom, from assisting numerous other teachers, and from running workshops, the first issues in organizing instruction using computers in the chemistry classroom are the number of computers available and their location.

In our opinion the best arrangement is to have three students to a computer. From our personal observations, a team of three students at the computer is to be preferred to individual use of the computer or two students per computer. The reasons for this magic number of three are multiple. The first is a matter of teacher supervision of students at the computer. With a class of twenty-four students, the sheer physical requirements on the teacher to monitor student progress become prohibitive if each is using a computer. This problem is even more severe than just ensuring that each student understands the assignment since at the introduction of new software there is a learning time during which the student must master the features and 
controls of the program. Teaming students in threes has proven to be a successful solution to this problem. Such teams can frequently teach themselves how to use the software and reduce the load on the teacher. Moreover, eight computers with teams of three are far more manageable for the teacher. Some of our colleagues have opted for two students per computer. However, in our experience, when using teams of two there is a greater likelihood that a dominant partner emerges and controls the computer.

Another argument for teams is that it is our experience that through group work students learn more. The teams engage in active discussions as they try to answers questions posed by the teacher.

A strategy that we have adopted for introducing students to new software is as follows. In class discussion we elicit from the students the parameters for the investigation. A list is made of these parameters and functionalities. Subsequently, the first task for the teams is to examine the software and find the controls for the parameters and the controls for the functionalities necessary for later investigations. This can be done as a guided discovery prior to a pre-introductory lecture, or after the introductory lecture.

We have found that the physical location of the computers has a great impact on their usage both for content instruction and for support in the formative assessment of student progress. By locating the computers in the classroom we are able to engage the students in learning logs and concept mapping on the computers. This would not be possible if the computers were located in an external "computer lab". The utility of learning logs and concept mapping on the computer is discussed below. Moreover, by having computers in our own classroom we maintain control over what is on the hard disk, the upgrading of system components, and their use for demonstration purposes. Many times in teaching, the "teachable" moment comes unexpectedly and immediate access to a specific teaching tool, such as the computer, can make the difference between making a point and allowing the moment to slip away.

\section{An Example of Using Software in the Chemistry Classroom}

The way we structure activities surrounding a piece of computer software so as to lead students to their own investigations and presentations can be exemplified by an exercise our class did using the Diatomic Explorer produced by the QSAD project. This activity is, in fact, the one that gave rise to the learning log on bonding quoted below. 
The class began with an assignment to use the Diatomic Explorer to find the equilibrium bond distance between the atoms in a polar covalent, non-polar covalent, and ionic molecule. Their questions relating to this assignment were written up as part the electronic journal each team is required to maintain on the computer. (These are the electronic learning logs discussed below.) After completing this assignment, each student team was asked to select an area of special interest for investigation. They were told that they would be expected to give a presentation to the class on their topic. Topics students selected included the variation of electronic density as a function of the atoms in the molecule and their location, bond energy versus bond length, bond energy as a function of orbital overlap, and so forth. Each team had to first show us the organization of their presentation in a matrix format. The matrix indicated what topics were to be covered and which students in the group would cover them. After we approved the idea for the investigation, each team used the software to answer their questions, and then presented their work to the class.

The concurrent use of learning logs, concept mapping (described below), and observation of the students in the classroom allowed us to triangulate our assessment of the students' progress. Based on this assessment we were able to provide well-motivated suggestions and support for the student investigations. As part of the constructivist approach we adopted, during the investigations we tried to avoid direct answers to questions we felt the students were capable of answering on their own. We did provide calculated hints and feedback on use of the software to assist their investigations. Closure on these investigations almost invariably occurred by the time of the teams' class presentations. At this time even if a misconception remained, either other students would point out the difficulty during the presentation, or through pointed questioning we were able to resolve the misconception.

As an example, one presentation addressed the idea that the charge density might shift between the atoms of different types. The students used the Diatomic Explorer (Figure 4) to compare the charge densities of different compounds.

Based on the visual displays, the students decided that the display of a symmetrical charge density was a better indicator of a nonpolar covalent bond than an electronegativity difference. In the following picture (Figure 5) the charge density is shifted toward the fluorine ion and the nucleus of the sodium ion is completely out of the electron cloud. 


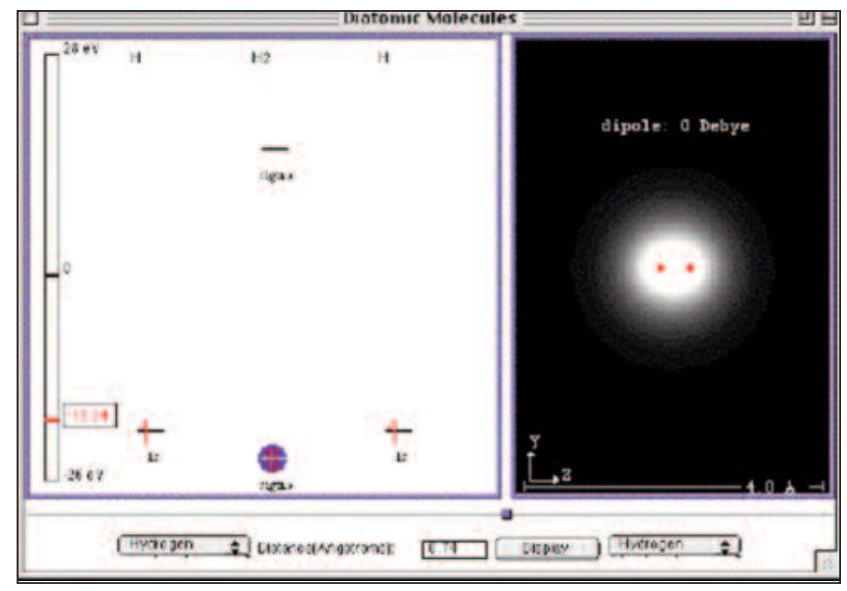

Figure 4. View of Computer Simulation: Diatomic Molecule Explorer. Two hydrogen atoms are shown covalently bonded.

\section{Formative Assessment and Evaluation with Computers}

\section{Learning Logs}

From our experience, one of the most significant affordances the computer offers is a very effective and efficient means for the teacher to monitor, pace, and guide students' progress. Interactive collaborative learning logs are continuous scripts between the teacher and student groups. Students, working in groups of three are required to draft a consensus

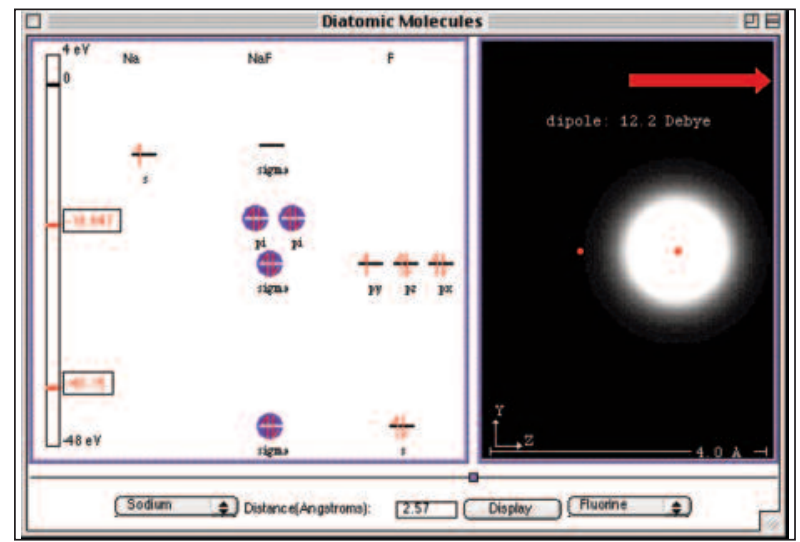

Figure 5. View of Computer Simulation: Diatomic Molecule Explorer. A sodium and a fluorine atom are shown bonded together. The large charge shift results in no electronic density around the sodium atom (based on the intensity level set here). 
opinion in response to teacher's questions. The group response generates further questions as the teacher probes the understanding of the threesome. In this way, the teacher has a chance to identify the group's beliefs that represent alternative conceptions. The teacher, acting as a mentor, can ask the next round of questions or suggest possible investigations that put the group on track toward a more fulfilling understanding of the concepts.

We instituted the process of learning logs to foster scientific understanding, to document the understanding and to monitor the changes in that understanding (Audet, Hickman \& Dobrynina, 1996). Groups date their entries, e-mail them to their teacher, and wait for responses to guide their investigation. In school systems that do not have electronic mail, the process can be handled by saving learning log files to floppy disk. Although this is not as efficient, it works very well. The teacher always responds in a bold Font, underlined, while the students are allowed to use any other Font. As with any other scientific notebook, the work is treated as if written in permanent ink and the students do not delete any entries, but add to them. In this way the learning log becomes a record of the growth and conceptual changes of the group.

While it is possible for students to maintain journals without computers, from our experience, and from what has been related to us by other teachers, such manual journals require far more work than learning logs maintained on a computer (Audet, Hickman and Dobrynina, 1996). Responding over a network to students' logs provides an immediacy in response which can be critical for student learning. Such immediacy is very difficult to maintain with handwritten documents.

The following example of a learning log entry is from a sophomore high school Honors Chemistry class. The questions relate to their preliminary study in the laboratory of atomic emission spectra.

Pre-lab: Explain what gives rise to the color visible to the naked eye, that is emitted by the gas discharge tube?

"Is dependent on the wavelength and amplitude emitted in photons by falls of electron. Increasing amplitude of a certain color the greater effect on the visible color."

Please write in complete sentences. Why do you think amplitude has the greatest effect on the color that we see?

"Amplitude has the greatest effect on the color that we see because it increases intensity, which is because a greater amplitude means a greater energy." 
To chart the growth of the group, questions are asked after the lab. Then the teacher publishes selected answers and distributes them to the class. The growth of the group becomes apparent when the complexity and accuracy of the answers provided in the following post-laboratory session are analyzed.

Post-lab: Explain what gives rise to the color emitted by the gas discharge tube?

"We believe that the color we see emanating from each gas discharge tube is a result of the individual colors of brightest intensity combining. The brightest intensities of color are the ones our eyes are most sensitive to, and therefore all the faintest colors are not visible to us."

Do you think that the faint colors have anything to do with what we see? How is brightness of color accounted for in quantum chemistry? "The light we see emitted from each gas discharge tube is the result of taking a weighted average from all individual spectral lines. This mixing of the individual wavelengths results in an average wavelength and this is what causes the light we see emitted by the tube by our naked eye."

"Intensity is a result of how many photons are emitted in our visible range. High intensity is due to more photons stimulating our eyes. These photons are released because the electron falls are more common. The number of photons are related to amplitude."

The group's thoughts are more complete and errors regarding amplitude, intensity, energy, and color have been corrected.

The following example of a learning log entry is from a different High School Honors Chemistry class. These are two questions from a student group prior to their investigations using computer simulations designed to study the concepts of quantum chemistry.

Pre-lab: Explain how bonding occurs from a quantum chemistry perspective.

"Bonding occurs when the energy of the molecule is less than the energies of the individual atoms after it bonds. The energy must be the most exothermic."

This is true, but you have not yet connected a quantum chemical explanation to the bonding of atoms. 
"Bonding occurs when atomic orbitals overlap with the molecular orbitals to form a more stable molecule."

I do not think that you mean, "The atomic orbitals overlap with the molecular orbitals." I need a more complete answer to determine exactly what you do mean.

We attempted to tailor the activities of the class by determining the level of student understanding as defined in these pre-lab communications. Although this group is beginning to use the terms from quantum science, they are still confusing the definitions and their relationships as they begin work on the software lab.

Post-lab: Explain bonding from a quantum chemistry perspective. "When two atoms are bonding they must be at a certain distance away from each other, where the waves associated with the valence orbitals interfere constructively with each other to form a large amplitude in between the nuclei. When squared this shows an increase in charge density. The positively charged nuclei are attracted to the electrons that are most probably there."

\section{Very good, but what stops the atoms from getting even closer?}

"The atoms can't get any closer because the protons in the nucleus will repel each other. Also, the large amount of constructive interference between the waves associated with the valence orbitals, of the bonding atoms, occur only at one distance between the atoms. As the atoms get closer or farther away however, there is destructive interference or less constructive interference, which results in less of a charge density. So if the atoms bond at the extremes the net force of attraction is less between protons and electrons, and therefore the bond is not as strong and the molecule not as stable."

After the activity the group has a more accurate scientific understanding of atomic and molecular orbitals, interference, energy, and the forces that hold molecules together. 


\section{Concept Mapping}

As we have seen above, students develop alternative conceptions regarding quantum science in chemistry. In our classes, students are encouraged to produce concept maps with which we can identify gaps in their learning and to help them understand the consequences of these gaps. The concept map, as defined by Novak, is composed of two fundamental components: the concepts that are placed hierarchically on the map, and the linking phrase between a pair of components which creates a proposition (Novak \& Gowin, 1984). There are other kinds of maps which can be constructed which link concepts. However, it is our research experience, as well as that of many other researchers, that providing these constraints results in a map of a student's comprehension of the subject which best provides the student with a study aid, and the teacher or researcher with a window into the student's thinking.

While students can use the concept maps to identify weaknesses in their understanding, the maps can be used by the teacher as a means for formative assessment so that adjustments can be made to the teaching and learning process. Even without practice in evaluation, one can look at the initial concept map (Figure 6) and see gaps in student understanding.

The links that are present are minimalist phrases that are open to multiple interpretations. To know what one student actually means by a map, the teacher must engage the student in a conversation in which the student

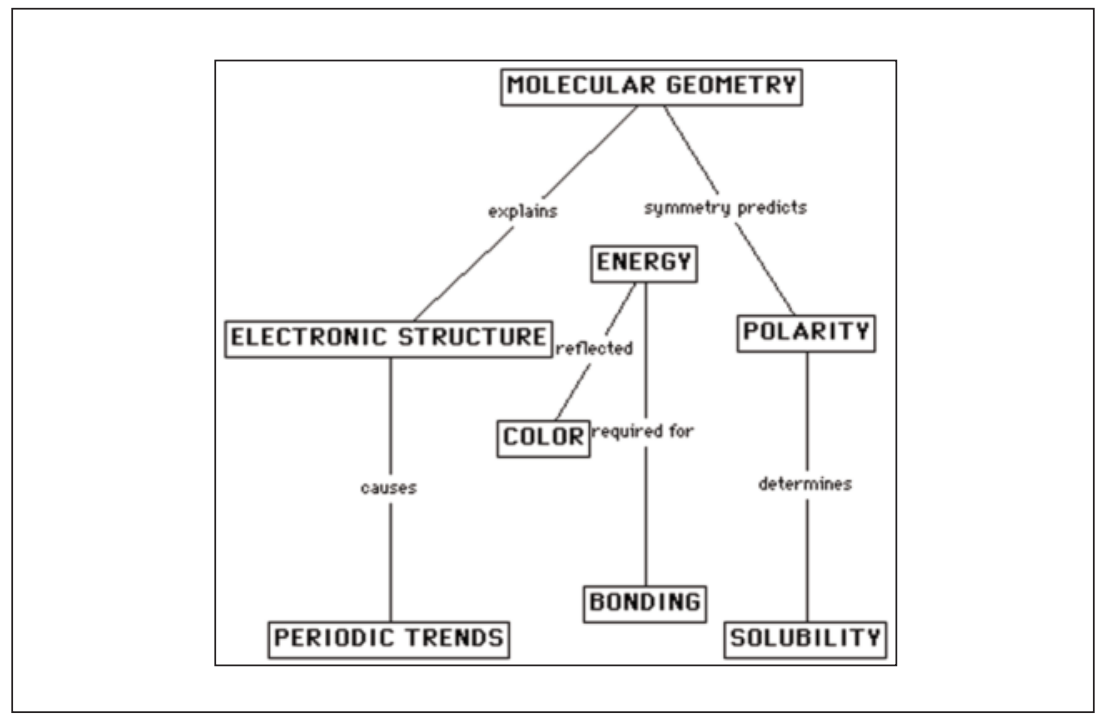

Figure 6. Initial concept map of student \#1 undergoing intervention. 
explains the linking phrases. For example, it is true that polarity is connected to solubility, but does the student have the ability to show how this is determined? During the unit based on the QSAD software, students study the relationships between these concepts using computer simulations. If meaningful learning occurs, students respond by connecting the new concepts to their core understanding. The final concept map (Figure 7) below shows obvious improvement for the same student.

An examination of the map shows that the student's weakest areas are related to the connections between color and electronic structure. In fact, the whole topic of electronic transitions and the release of quantized energy in the form of electromagnetic spectra remains vague in the student's map. This is understandable since the parts of the simulation related to spectroscopy were not a part of this particular exercise. However, this map is approaching the complexity of the two dimensional expert map in Figure 8 on the following page.

\section{The Computer Supporting Metacognitive Activities by the Students}

In How People Learn: Bridging Research and Practice (National Research Council, 1999) the Committee of the National Research Council points out the importance of helping students develop learning strategies. Both concept maps and learning logs are such metacognitive activities for the students. It is possible to make concept maps, and for students to engage in group and journal keeping activities, without the computer. However, in this case the computer proves to be an enabling technology that makes the use of learning logs and concept maps practical for classroom use. In the

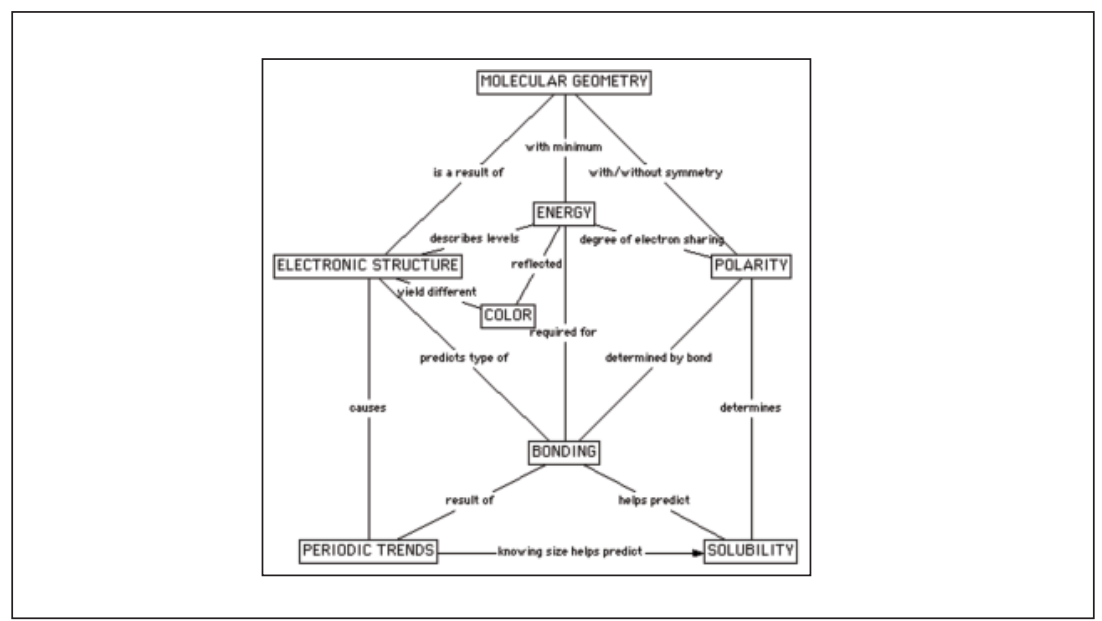

Figure 7. Final concept map of student \#1 after the intervention. 


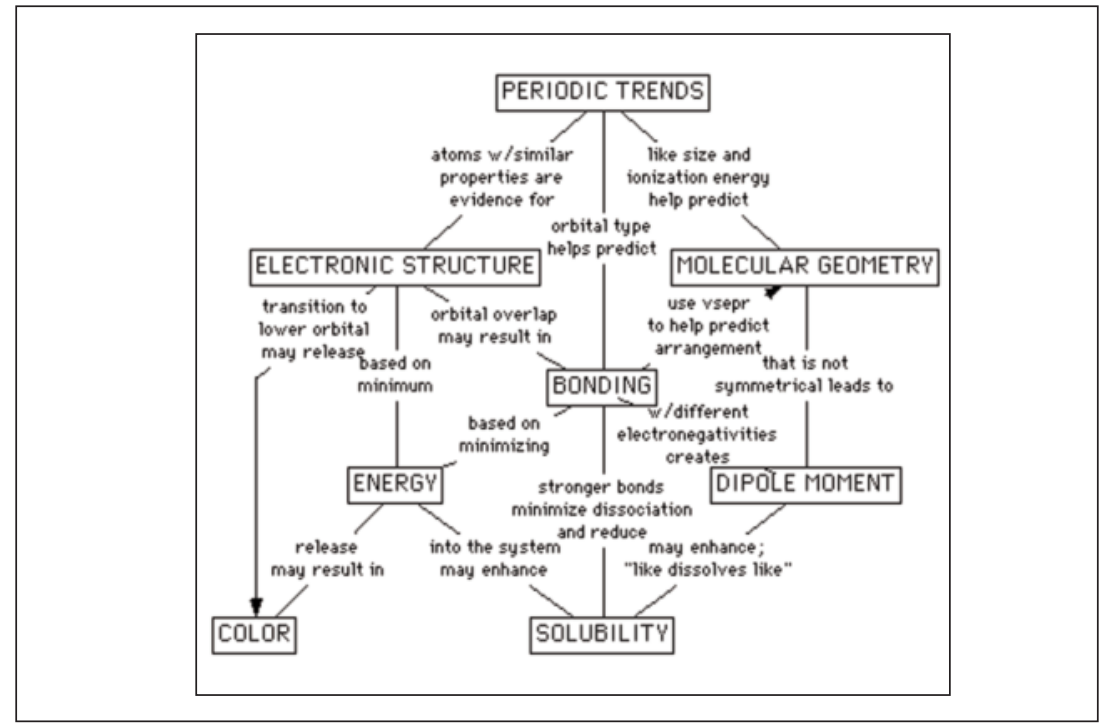

Figure 8. Expert concept map by Honors Chemistry teacher.

absence of the computer, learning logs become too much of a burden for rapid turnaround by the teacher for effective formative assessment. Similar problems arise with concept maps. The ease of communication between student and teacher makes practical these two effective tools for student learning and formative assessment.

\section{Outcomes from the Use of Concept Maps and Learning Logs}

It is our experience that students need convincing to take advantage of interactive collaborative learning logs and concept maps as tools to monitor their learning. With practice, these two activities provide students with an efficient method for monitoring their progress both on their own and through external feedback. When a student asks teammates how to make predictions regarding chemical behavior during a learning log entry, she has learned how to learn and work in a group, as well as possibly having learned a new concept. Similarly, through the use of a concept map, students can quickly determine which relationships they have most difficulty explaining. We have observed that the writing level of the students in the course gradually improves as the groups get better at explaining their reasoning. Similarly, as the course progresses, concept maps improve as new student understanding becomes connected to the individual's core knowledge. This is what is ultimately needed for meaningful student learning (Ausubel, 1963). 


\section{Conclusions}

Experimenting with abstract models of microscopic behavior was impossible at the high school level before computer simulations. With computers, students can both visualize and investigate the consequences of the rules of the model for a system. Students get a chance to learn science by doing "real science," and they are given the chance to test their own theories and benefit from meaningful learning as an outcome of their inquiries.

The kind of computer simulations referred to above have helped us, and the teachers we work with, to in-part resolve the dilemma of how to foster good investigatory habits among our students. Certainly in chemistry the software developed by the WAMNet and QSAD projects enable students to carry out their own investigations. Previously, without the technology, it simply was not possible to provide students with the apparatus to investigate the parameter ranges which so frequently define a scientific theory, i.e., the extreme ranges which are so hard to achieve in the laboratory.

Our research has also found that using the technology has an impact on students' judgment. Professional researchers develop their intuition for their area of expertise from their intense contact with the field. With the computer models we have discussed, our students stay after school spending hours studying things of interest. This has given them the insight and confidence to state their views both in-group and class discussions in a way that they had not done before.

Technology has also influenced equity in the classroom. We have coined the phrase "mouse control," referring to the control of the computer activity by the person in the group who possesses the mouse. If the teacher is careful to shift this control around, then power is distributed among the group members. This is often a new experience for female members of science classes, who may begin their group participation as less assertive than the males.

Finally, the introduction of computers into the classroom has provided teachers with a new window into the development of students' alternative conceptions. Through the use of concept maps and learning logs, teachers can engage in real-time formative assessment on a continuous basis. Indeed, formative assessment can be effectively integrated into the classroom routine. The use of these learning tools is double-edged as students in turn can use them to monitor their own progress. 
Overall, the use of computer models in our classroom has changed dramatically the content and way that we teach, students learn, and how we as teachers assess our classroom. Through the use of concept maps, learning logs, and computer simulations students obtain a deep level of understanding, avoid rote learning, and develop an intuition about physical phenomena.

\section{References}

Ausubel, D. P. (1963). The psychology of meaningful verbal learning. New York: Grune and Stratton.

Audet, R. H., Hickman, P. \& Dobrynina, G. (1996). Learning logs: a classroom practice for enhancing scientific sense making. Journal of Research in Science Teaching. Vol. 33 , issue 2, pp. 205-222.

Collins, A., Brown, J. S. \& Holum, A. (1991). Cognitive apprenticeship: making thinking visible. American Educator. pp. 15-28.

Gabel, D. L. \& Bunce, D. M. (1994). Research on problem solving in chemistry. Handbook of Research on Science Teaching and Learning. New York: Simon and Schuster. pp. 301-326.

Hewson, M. G. \& Hewson, P. W. (1983) Effect of Instruction Using Students' Prior Knowledge and Conceptual Change Strategies on Science Learning. Journal of Research in Science Teaching. Vol. 20, issue 8, pp. 731-743.

Kuhn, Thomas (1970). The Structure of Scientific Revolutions. Second Edition, Enlarged. Chicago: The University of Chicago Press.

National Research Council (1996). National Science Education Standards. Washington, DC: National Academy Press.

National Research Council (1999). How People Learn: Bridging Research and Practice. Washington, DC: National Academy Press.

Novak, J. \& Gowin, B. (1984). Learning how to learn. Cambridge, England. Cambridge University Press. pp. 1-34.

Posner, G., Strike, K., Hewson, P., \& Gertzog, W. (1982). Accommodation of scientific conception: Toward a theory of conceptual change. Science Education, 66, pp. 211-227. 